\title{
Perspectivas da Pesquisa Prospectiva com Bebês Irmãos de Autistas
}

\author{
Perspectives of prospective research of younger \\ siblings of children with autism
}

Perspectivas de la pesquisa prospectiva con bebés hermanos de autistas
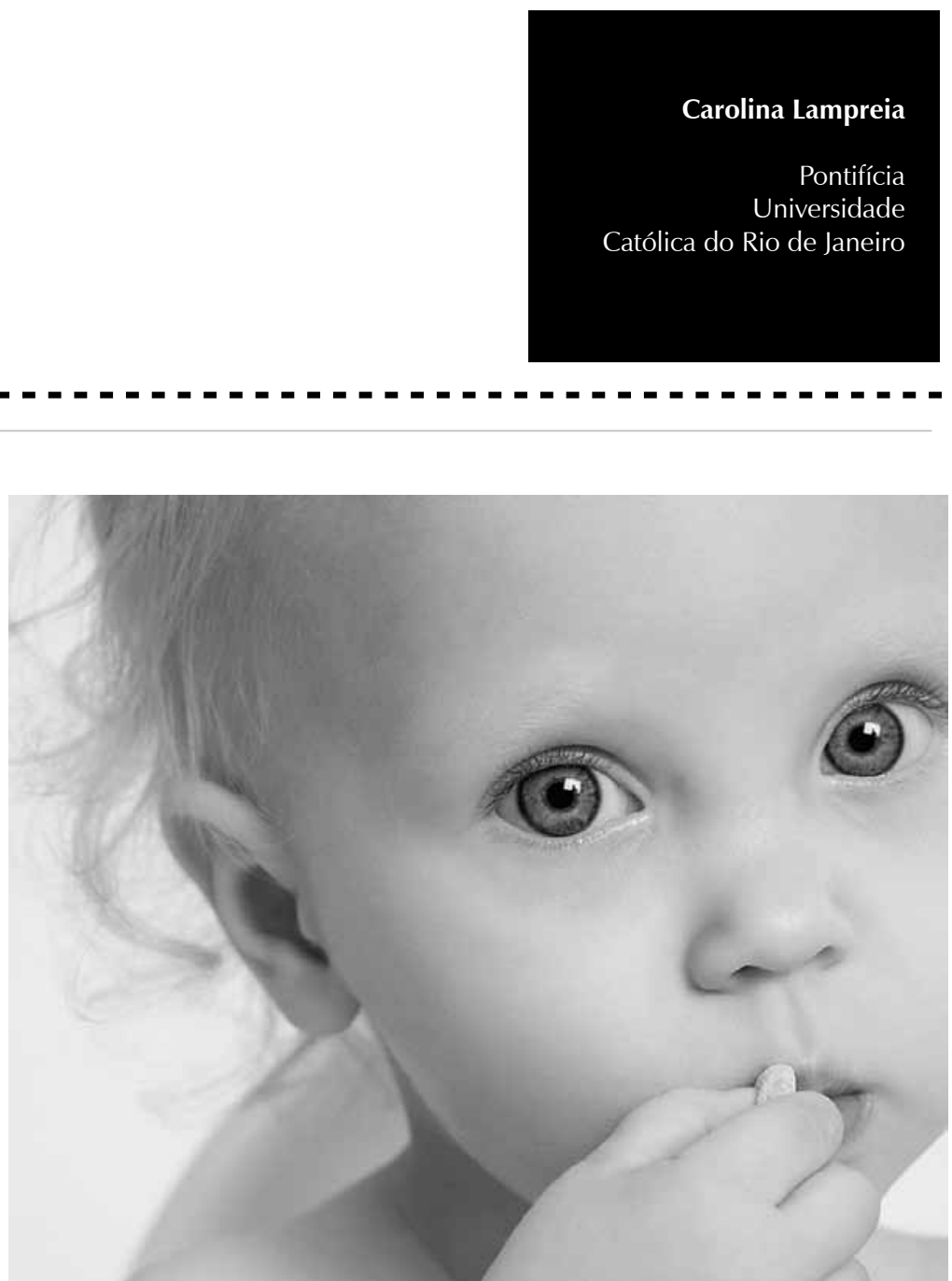
Resumo: A identificação precoce de bebês com risco de desenvolver um quadro autístico é importante por permitir uma intervenção também precoce, minimizar o sofrimento da família e tornar os profissionais de saúde e educação mais vigilantes para os possíveis sinais de risco de autismo. Ao longo dos últimos anos, tem havido um interesse crescente na pesquisa sobre indicadores precoces do autismo. O objetivo deste trabalho é discutir uma metodologia longitudinal prospectiva para a identificação de sinais precoces de risco do autismo, a partir de estudos envolvendo bebês irmãos de autistas, que também permita investigar a identificação de subtipos de autismo e proporcionar melhor entendimento da relação entre a tríade de prejuízos que o define. Serão apresentados, inicialmente, os diferentes tipos de estudos disponíveis sobre indicadores de risco de autismo - relatos dos pais, estudos de vídeos familiares e de atenção compartilhada, instrumentos disponíveis e pesquisas prospectivas de bebês irmãos de autistas.

Palavras-chave: Autismo. Pesquisa prospectiva. Identificação precoce. Subtipos.

Abstract: Early identification of infants at-risk of autism is important for early intervention, to minimize the family suffering and to allow professional surveillance of possible signs of autistic risk. Lately the literature on autism has witnessed a growing interest in this area of research. The aim of the present paper is to discuss a prospective longitudinal methodology for the identification of early signs of autism through studies with younger siblings of children with autism that could also allow the investigation of subtypes of autism and a better understanding of the triad of impairments that define autism. At first, different kinds of studies about early signs of autism will be presented - parents' reports, studies of family home videos and joint attention, available instruments and prospective research of younger siblings of children with autism.

Keywords: Autism. Prospective research. Early identification. Subtypes.

Resumen: La identificación precoz de bebés con riesgo de desarrollar un cuadro autístico es importante por permitir una intervención también precoz, minimizar el sufrimiento de la familia y tornar los profesionales de salud y educación más vigilantes para las posibles señales de riesgo de autismo. A lo largo de los últimos años, ha habido un interés creciente en la pesquisa sobre indicadores precoces del autismo. El objetivo de este trabajo es discutir una metodología longitudinal prospectiva para la identificación de señales precoces de riesgo del autismo, desde estudios con bebés hermanos de autistas, que también permita investigar la identificación de subtipos de autismo y proporcionar mejor entendimiento de la relación entre la tríade de perjuicios que lo define. Serán presentados, inicialmente, los diferentes tipos de estudios disponibles sobre indicadores de riesgo de autismo - relatos de los padres, estudios de vídeos familiares y de atención compartida, instrumentos disponibles y pesquisas prospectivas de bebés hermanos de autistas.

Palabras clave: Autismo. Pesquisa prospectiva. Identificación precoz. Subtipos.

O autismo é concebido hoje como um transtorno do desenvolvimento de base biológica inata, embora sua etiologia ainda permaneça desconhecida. Caracterizase por uma tríade de prejuízos nas áreas de interação social e de comunicação e por padrões restritos e repetitivos de comportamentos, interesses e atividades, mas abrange um spectrum muito heterogêneo de quadros clínicos ou comportamentais.

Apesar de, desde Kanner (1943), serem mencionados sinais muito precoces do autismo, tais como a falta de ajustamento postural, de contato ocular e de movimentos antecipatórios, ele costuma ser oficialmente diagnosticado por volta dos três anos de idade, através da CID-10 (Classificação Internacional de Doenças) e do DSM-IV-TR (Diagnostic and Statistical Manual of Mental Disorders-Revised), instrumentos elaborados respectivamente pela Organização Mundial de Saúde (WHO, 1998) e pela Associação Americana de Psiquiatria (APA, 2002). Contudo, nos últimos anos, vem sendo enfatizada a necessidade de uma identificação precoce de bebês que apresentam risco de desenvolver um quadro autístico, visando a uma intervenção também precoce que procure recuperar ou minimizar os efeitos do transtorno autístico. Embora ainda não seja possível garantir que um programa de intervenção precoce, antes dos cinco anos de idade, irá reverter o quadro do autismo, 
alguns autores consideram que há evidências crescentes de que a intervenção traz melhoras no quadro clínico dos transtornos do espectro autístico (TEAs) (Charman \& Baird, 2002). Por essa razão, na última década, tem sido enfatizada de forma crescente a importância da diminuição da idade de diagnóstico do autismo para os 18 meses de idade, havendo até pesquisas que indicam que ele poderia ser feito antes, entre 8 e 12 meses.

A identificação precoce também parece ser importante no sentido de procurar minimizar o sofrimento da família e de tornar os profissionais de saúde e educação mais vigilantes para os possíveis sinais de risco autístico. Além disso, ela também poderá permitir a identificação de subtipos de autismo, isto é, de diferentes perfis e graus de severidade, assim como um melhor entendimento da tríade de prejuízos.

Os itens de avaliação tanto da CID-10 como do DSM-IV-TR são muito genéricos e não permitem tal reconhecimento precoce. Por essa razão, ao longo dos anos, vários pesquisadores têm procurado desenvolver instrumentos mais específicos, precisos e exaustivos. Eles podem ter a forma de entrevistas, questionários ou protocolos de observação, mas são poucos os instrumentos que se destinam a uma avaliação precoce. Os principais, por apresentarem estudos de validade e serem os mais utilizados, são o ADI-R (Lord, Rutter, \& Le Couteur, 1994) - uma entrevista a ser feita com o cuidador da criança a partir dos 18 meses até a idade pré-escolar - e o CHAT (Baron-Cohen, Allen, \& Gillberg, 1992), que deve ser aplicado aos 18 meses de idade e consiste em um questionário de nove perguntas a serem feitas ao cuidador da criança e de cinco itens de observação para orientar o pediatra durante a consulta.

A questão é que esses dois instrumentos só permitem a identificação de risco de autismo a partir dos 18 meses, embora haja outras fontes de informação sobre indicadores ainda mais precoces do autismo. São elas: o relato retrospectivo dos pais, a análise de vídeos familiares e pesquisas sobre comportamentos de atenção compartilhada, isto é, sobre a comunicação social pré-verbal no desenvolvimento típico. Os estudos de vídeos familiares têm sido capazes de encontrar sinais indicativos de risco autísticos já aos 12 meses de idade (Adrien et al., 1991; Baranek, 1999; Osterling \& Dawson, 1994; Osterling, Dawson, \& Munson, 2002). Além disso, alguns autores, seguindo um enfoque desenvolvimentista, têm considerado que, dado o prejuízo de cunho biológico, o quadro clínico apresentado pela criança autista pré-escolar se deve à impossibilidade em desenvolver interações sociais/comunicativas típicas da fase de bebê (Dawson \& Galpert, 1986; Dawson \& Lewy, 1989; Hobson, 2002; Trevarthen, Aitken, Papoudi, \& Robarts, 1998). Tem sido enfatizada, então, a necessidade de maior compreensão do desenvolvimento típico da comunicação não-verbal, mais especificamente da atenção compartilhada, que se desenvolve entre os 9 e 15 meses, isso porque ela tem sido vista como precursora ou suporte para o desenvolvimento normal da fala que está prejudicada no autismo (BristolPower \& Spinella, 1999).

Desde 2005, pesquisas longitudinais prospectivas têm procurado indícios de risco de autismo a partir dos 6 meses de idade. Para esse fim, têm sido observados bebês com irmãos mais velhos com diagnóstico de autismo, tendo em vista uma recorrência $20 \%$ maior de autismo nessa amostra do que na população em geral (Sumi, Taniai, Miyachi, \& Tanemura, 2006, conforme citado por Merin, Young, Ozonoff, \& Rogers, 2007). Esses estudos têm utilizado, principalmente, categorias quantitativas discretas de atenção compartilhada - olhar para o experimentador, alternância do olhar, apontar e seguir o apontar - que envolvem interações triádicas 
mãe-bebê-objeto e se desenvolvem entre os 9 e 15 meses de idade. Elas são consideradas precursoras da linguagem e marcadoras do autismo (Bosa, 2002). Contudo, é importante observar que, do ponto de vista do desenvolvimento, essas categorias também têm seus precursores, que devem ser encontrados nas interações diádicas mãe-bebê, entre o nascimento e os nove meses de idade. É nesse período inicial do desenvolvimento que começam a se desenvolver as habilidades comunicativas diádicas básicas - contingência, reciprocidade, antecipação, alternância de turno - sem as quais não há diálogo, mas, para que isso ocorra, é preciso que o bebê seja capaz de engajamento afetivo, é preciso que ele seja sensível e responsivo às expressões emocionais dos outros, o que parece não ocorrer com bebês com risco de autismo (Hobson, 2002). Esse aspecto afetivo, qualitativo, do desenvolvimento tem sido bastante investigado em pesquisas de Psicologia do desenvolvimento, que, na década de 1970, desenvolveu uma metodologia específica de microanálise de vídeos de interação mãebebê (Lewis \& Rosenblum, 1974; Schaffer, 1977). No entanto, ele tem sido praticamente desconsiderado em estudos sobre autismo.

O objetivo deste trabalho é discutir uma metodologia longitudinal prospectiva para a identificação de sinais precoces de risco do autismo a partir de estudos que envolvem bebês irmãos de autistas que também permita investigar a identificação de subtipos de autismo e proporcionar melhor entendimento da relação entre a tríade de prejuízos que o define. Inicialmente, serão apresentados os diferentes tipos de estudos disponíveis sobre indicadores de risco de autismo - relatos dos pais, estudos de vídeos familiares e de atenção compartilhada, alguns instrumentos disponíveis e pesquisas longitudinais prospectivas de bebês com irmãos autistas, mais recentes.

\section{Estudos sobre a identificação precoce}

Charman e Baird (2002) afirmam ser um consenso o fato de que o diagnóstico de TEA é estável aos 2 e 3 anos de idade. Várias pesquisas têm procurado avaliar a possibilidade de uma identificação precoce, confiável e estável, do autismo aos 2 anos de idade, e têm concluído de maneira afirmativa (ver, por exemplo, Eaves \& Ho, 2004; Lord, 1995; Stone et al., 1999). Esses estudos têm apontado falhas em comportamentos de atenção compartilhada aos 2 anos como os melhores discriminadores do autismo. Lord (1995) cita a falta de procura para compartilhar alegria, de atenção à voz do adulto, do apontar para dirigir a atenção, e Stone et al. (1999) mencionam déficits no uso de comportamentos comunicativos não-verbais, a falta de reciprocidade social ou emocional e o atraso na aquisição da fala. Essas mesmas falhas foram encontradas em um estudo de Wetherby et al. (2004).

Os diferentes tipos de estudos focados na busca de indicadores precoces de risco de autismo envolvem relatos dos pais, análises de vídeos familiares, comportamentos de atenção compartilhada, instrumentos de identificação precoce e estudos longitudinais prospectivos de bebês com irmãos autistas.

\section{Relato dos pais}

Um estudo de Chawarska e Volkmar (2005), que aborda os sintomas precoces do autismo, afirma que 30 a $54 \%$ dos pais de crianças diagnosticadas com autismo tiveram as primeiras preocupações antes dos 12 meses, e 80 a $90 \%$ reconheceram as anormalidades aos 24 meses.

Estudos de relatos retrospectivos dos pais sugerem que os sintomas precoces devem ser encontrados em déficits de habilidades sociais emergentes, tais como antecipação 
ao ser pego, demonstração de afeto para pessoa familiar, interesse em crianças, tentar alcançar pessoa familiar e jogos simples de interação com outros. Nos primeiros 6 a 8 meses, por exemplo, os bebês afetados apresentaram atenção visual a pessoas diminuída, procuraram menos as pessoas e se engajaram menos em intercâmbios sociocomunicativos através do sorriso e da vocalização. Além disso, os primeiros sinais podem incluir dificuldades de regulação da excitação (arousal), mas os autores consideram que a evidência direta sobre o quadro clínico, nessa idade, é muito limitada, apesar dos relatos precoces dos pais.

Em outro estudo, Chawarska et al. (2007) investigaram a idade de surgimento dos primeiros sinais de autismo através do relato dos pais. Identificaram três grupos segundo a idade do início de suas preocupações: o primeiro entre 0 e 10 meses de idade, o segundo, entre 11 e 17 meses, e o terceiro, depois dos 18 meses. As principais preocupações dependeram da época de reconhecimento pelos pais. No primeiro grupo, elas incluíram o atraso no sorrir e andar. Os autores consideram que os fatores patogênicos responsáveis pela disfunção social podem estar presentes desde o nascimento, embora a severidade dos sintomas e o padrão temporal da expressão possa variar. Isso poderia fazer com que os pais começassem a se preocupar em momentos diferentes e talvez por razões diferentes.

\section{Estudos de vídeos familiares}

A estratégia adotada para se conhecer os primeiros sinais indicativos de risco autístico tem sido a análise retrospectiva de vídeos dos primeiros meses de vida de crianças já diagnosticadas como portadoras de autismo realizados pela própria família, em situações informais como, por exemplo, a festa do primeiro aniversário, brincadeiras, banho
(Adrien et al., 1991; Adrien et al., 1993; Baranek, 1999; Osterling \& Dawson, 1994; Osterling et a., 2002). Esses estudos têm procurado identificar categorias que, aos 12 meses de idade ou mesmo antes, permitam discriminar crianças com risco de autismo ou TEA de crianças com desenvolvimento típico e retardo mental. Mas é importante ressaltar as limitações metodológicas de tais estudos, tendo em vista o fato de que as filmagens não foram realizadas com esse objetivo.

Dois estudos de vídeos familiares franceses (Adrien et al., 1991; Adrien et al., 1993; Baranek, 1999; Osterling \& Dawson, 1994; Osterling et al., 2002)avaliaram vídeos familiares nos dois primeiros anos de vida, através da escala francesa ERC-N desenvolvida para a avaliação do desenvolvimento típico. Ela apresenta seis categorias funcionais mais gerais: socialização, comunicação, adaptação ao ambiente, tato-tonus-motilidade, reações emocionais e instintivas, atenção e percepção. Os autores desses dois estudos concluem que os seguintes 12 comportamentos foram incluídos como fator de risco de autismo: ignora pessoas, prefere isolamento, interação social pobre, sem contato ocular, sem sorriso social, falta de expressões faciais apropriadas, falta de gestos apropriados e/ou posturas expressivas, comportamento estereotipado, posturas não-usuais, sem expressão de emoções, contato ocular anormal, atenção instável/distração fácil.

Outro estudo explorou a utilidade de medidas sensório-motoras, além dos comportamentos sociais, como preditores precoces do autismo, já que cerca de 70 a $80 \%$ dos autistas apresentam disfunções sensoriais (Baranek, 1999). A autora comparou os vídeos de crianças autistas (AU), crianças com atraso de desenvolvimento (AD) e com desenvolvimento típico (DT), de 9 a 12 meses de idade. Quatro categorias permitiram diferenciar as autistas dos outros dois grupos: maior freqüência de objetos na boca, mais ocorrências de aversão ao toque social, menos orientação 
para estímulos visuais e mais instigações para responder ao seu nome.

O crescente consenso quanto à falha no desenvolvimento da comunicação nãoverbal e, mais especificamente, à falha dos comportamentos de atenção compartilhada como um dos possíveis marcadores do autismo requer a revisão de alguns estudos dessa área no desenvolvimento típico.

\section{Os comportamentos de atenção compartilhada}

Os diferentes comportamentos de atenção compartilhada - o engajamento conjunto, o seguir o olhar e o apontar, o apontar imperativo e o declarativo - têm sido vistos como um marco fundamental do desenvolvimento da criança por serem precursores do desenvolvimento da fala. Eles surgem entre os 9 e 15 meses de idade, quando mãe e bebê, em interações triádicas (mãe-bebê-objeto), começam a compartilhar o interesse por objetos. Antes disso, sua interação é apenas diádica (mãe-bebê), ou seja, mãe e bebê apresentam interações face a face e protoconversações, mas sem referência a objetos. Trevarthen et al. (1998) denominam essas duas fases de intersubjetividade secundária e intersubjetividade primária, respectivamente.

Vários estudos têm argumentado haver uma relação entre os comportamentos de atenção compartilhada e o desenvolvimento simbólico (ver Adamson \& McArthur, 1995; Baldwin, 1995; Bono, Daley, \& Sigman, 2004; Carpenter, Nagell, \& Tomasello, 1998; Tomasello, 1995; Tomasello, 2003; Tomasello \& Farrar, 1986). Por outro lado, vários estudos têm apontado os prejuízos de atenção compartilhada no autismo (ver Adamson \& McArthur, 1995; Carpenter \& Tomasello, 2000; Charman, 2004; Dawson et al., 2002; Sigman \& Kasari, 1995). Sigman \& Kasari, por exemplo, afirmam que crianças autistas olham menos para o experimentador, apresentam menor alternância do olhar, apontar e seguir o apontar do que crianças com desenvolvimento típico ou crianças com déficit mental.

Com base nos estudos de relatos de pais, vídeos familiares e atenção compartilhada, assim como no que se conhece do desenvolvimento típico de bebês, foram desenvolvidos instrumentos para a identificação precoce do autismo.

\section{Alguns instrumentos de identificação precoce do autismo}

Existem vários instrumentos para a identificação precoce do autismo por volta dos 2 anos idade, ou mesmo antes, mas apenas alguns serão revistos aqui : CHAT, M-CHAT, STAT e CSBS DP/SORF. O critério adotado para sua inclusão foi a brevidade de sua aplicação, a não exigência de treinamento, assim como os estudos de validação sobre as categorias indicativas de risco de TEA.

O CHAT (Checklist for Autism in Toddlers) (Baron-Cohen et al., 1992) é um instrumento de rastreamento a ser aplicado a toda a população, na consulta pediátrica de rotina aos 18 meses de idade. Ele inclui um questionário de 9 perguntas para os pais e 5 itens de observação para o profissional de saúde. Tanto no questionário quanto na observação, são avaliados: o jogo de faz-de-conta, o apontar protodeclarativo e o desenvolvimento intelectual. Apenas no questionário, são avaliados: o interesse social por outras crianças, o desenvolvimento motor, a brincadeira social de esconde-esconde, a atenção compartilhada através do mostrar e o apontar protoimperativo, e, apenas na observação, são avaliados: a interação social através do contato ocular e o seguir o apontar. O M-CHAT (Modified Checklist for Autism 
Reznick, Baranek, Reavis, Watson,

$\&$ Crais (2007) criaram um questionário para pais - 0 instrumento do primeiro ano (first year instrument - FYI), no qual cada pergunta se refere a um comportamento que tem sido associado a algum grau de eventual diagnóstico de autismo. $\mathrm{O}$ objetivo de longo prazo dos autores

é desenvolver uma ferramenta de rastreamento eficaz para pesquisadores, pediatras, clínicos e pais com o objetivo de identificar bebês de 12 meses com risco de autismo. in Toddlers) (Robins, Fein, Barton, \& Green, 2001), que também é um instrumento de rastreamento, inclui apenas o questionário do CHAT mais 13 itens que abrangem funções indicadas como responsáveis pelo autismo em outros estudos, e deve ser aplicado aos 24 meses de idade com o intuito de identificar também crianças com possível regressão entre os 18 e 24 meses.

O STAT (Screening Tool for Autism in Twoyear-olds) (Stone, Coonrod, \& Ousley, 2000) é uma medida interativa breve desenvolvida para ser usada por profissionais da saúde a fim de identificar crianças com necessidade de um teste de diagnóstico mais extenso e especializado. Serve como um segundo estágio do rastreamento, isto é, para diferenciar autistas de crianças com outros transtornos do desenvolvimento. $\mathrm{O}$ instrumento inclui 12 itens: 4 de imitação motora, 2 de jogo convencional e 4 de comunicação, como dirigir a atenção. Os autores afirmam que o STAT é provavelmente mais útil como instrumento de rastreamento de crianças menores, porque os itens avaliados têm mais probabilidade de estar presentes em idades mais jovens.

O CSBS DP (Communication and Symbolic Behavior Scales - Developmental Profile) (Wetherby et al., 2004) foi concebido como uma avaliação para o atraso da comunicação, mas ele pode ser útil para a identificação precoce de crianças TEA muito pequenas, porque avalia habilidades pré-lingüísticas identificadas como déficits em pré-escolares com TEA. É uma ferramenta estandardizada para o rastreamento e a avaliação de habilidades simbólicas e de comunicação de crianças entre 12 e 24 meses de idade, que inclui 3 medidas: 1) uma lista de checagem a ser completada pelos pais (Infant-toddler Checklist), 2) um questionário para o cuidador no follow-up (Caregiver Questionnaire), 3) uma avaliação da interação da criança com a mãe e o clínico, filmada (Behavior Sample), que inclui 7 habilidades pré-lingüísticas: emoção, olhar, comunicação, gestos, sons e palavras, compreensão e uso de objeto. Há boas evidências para sua confiabilidade e validade.

A partir dos vídeos do Behavior Sample, foi desenvolvido um instrumento que indica se há necessidade de se fazer um diagnóstico para TEA - o SORF (Systematic Observation of Red Flags for ASD). Esse instrumento inclui 29 itens, derivados dos critérios de diagnóstico para TEA e pesquisas com crianças pequenas com TEA, agrupados em 5 áreas: interação social recíproca, gestos não-convencionais, sons e palavras nãoconvencionais, comportamentos repetitivos e interesse restrito e regulação emocional.

A utilização do CHAT, do CSBS DP/SORF e do STAT aponta 12 categorias discriminativas. Destas, a categoria "protodeclarativo" é a única presente nos três instrumentos. As demais categorias - jogo de faz-deconta, seguir o apontar, imitação, interesse social/crianças, resposta ao nome, jogo convencional, contato ocular, expressão calorosa, coordenação do olhar/gesto/som, prosódia e movimentos repetitivos - estão presentes em apenas um instrumento.

Mais recentemente, tem havido interesse em desenvolver instrumentos de identificação ainda mais precoce do autismo, aos 12 meses de idade. Reznick, Baranek, Reavis, Watson e Crais (2007) criaram um questionário para pais - o instrumento do primeiro ano (first year instrument - FYI), no qual cada pergunta se refere a um comportamento que tem sido associado a algum grau de eventual diagnóstico de autismo. O objetivo de longo prazo dos autores é desenvolver uma ferramenta de rastreamento eficaz para pesquisadores, pediatras, clínicos e pais com o objetivo de identificar bebês de 12 meses com risco de autismo. Ele foi desenvolvido a partir de comportamentos-alvo identificados 
em estudos retrospectivos e prospectivos, e abrange dois domínios de desenvolvimento: o da comunicação social e o das funções sensoriais-regulatórias. O primeiro domínio inclui itens sobre a orientação social e a comunicação receptiva, o engajamento afetivo, a imitação e a comunicação expressiva, e o segundo inclui itens sobre processamento sensorial, padrões regulatórios, reatividade e comportamentos repetitivos. Os autores observam, contudo, que a maioria dos comportamentos que indicam risco aos 12 meses não preocupam necessariamente os pais.

\section{Estudos longitudinais de bebês com irmãos autistas}

Os estudos vistos até aqui - relatos dos pais, vídeos familiares, atenção compartilhada e instrumentos específicos - são estudos retrospectivos que visam à identificação precoce do autismo entre 12 e 24 meses de idade. A partir de 2005, um grupo significativo de pesquisadores têm privilegiado estudos prospectivos, investigando longitudinalmente bebês com alto risco de autismo, isto é, com irmãos que já receberam o diagnóstico de autismo, já que esses bebês têm maior probabilidade de apresentar características do espectro do que bebês com irmãos sem esse diagnóstico.

Em 2007, foi publicado um número especial do Journal of Autism and Developmental Disorders com resultados preliminares de algumas dessas pesquisas (Bryson et al., 2007; Cassell et al., 2007; Gamliel, Yirmiya, \& Sigman, 2007; Iverson \& Wozniak, 2007; Loh et al., 2007; Merin et al., 2007; Presnames, Walden, Stone, \& Yoder, 2007; Sullivan et al., 2007). A metodologia utilizada por esses estudos está sintetizada na Tabela 1.

Tabela 1. Metodologia de estudos com bebês de alto-risco de autismo.

\begin{tabular}{llll}
\hline 8 Estudos & $\begin{array}{l}\text { Início avaliação } \\
\text { (meses) }\end{array}$ & $\begin{array}{l}\text { Periodicidade } \\
\text { das avaliações }\end{array}$ & $\begin{array}{l}\text { Categorias } \\
\text { avaliadas }\end{array}$ \\
\hline 13 & 5 & mensal (entre 5 e $14 \mathrm{~ms})$. & motor + vocalização (vídeo) \\
10 & 6 & bimestral (entre 6 e $18 \mathrm{~m}$ ) & afeto, atenção compartilhada \\
1 & 6 & semestral (entre 6 e $36 \mathrm{~ms})$. & atenção/fixação visual \\
14 & 4 & dez meses (entre 4 e $54 \mathrm{~ms}$ ) & $\begin{array}{l}\text { cognição, linguagem } \\
\text { atenção/fixação visual, afeto }\end{array}$ \\
9 & 6 & uma vez aos $6 \mathrm{~ms}$. & estereotipias (vídeo) \\
2 & 12 & 12 e $18 \mathrm{~ms}$. & atenção compartilhada \\
3 & 14 & aos $14 \mathrm{~ms}$. & atenção compartilhada \\
11 & 12 & aos $12 \mathrm{~ms}$. &
\end{tabular}

Cinco dos oito estudos iniciaram as avaliações antes dos 12 meses de idade. Apenas os estudos 13 e 10 fizeram um acompanhamento mais regular - mensal ou bimestral - dos bebês. Os demais fizeram observações/avaliações quando muito semestrais (estudo 1), sendo que a maioria o fez apenas uma ou duas vezes. A principal forma de avaliação foi a testagem, pois apenas dois estudos realizaram análise de vídeos. Finalmente, todos os estudos utilizaram categorias discretas em detrimento de categorias diádicas e/ou afetivas.

\section{Perspectivas da pesquisa longitudinal prospectiva}

A pesquisa prospectiva com bebês com irmãos autistas abre novas perspectivas para a identificação precoce de sinais de risco de autismo. Ela também pode se constituir em um valioso instrumento 
1 Para uma discussão mais ampla desses enfoques, ver Lampreia (2004).

2 Para uma descrição geral do desenvolvimento do nascimento até a formação do símbolo, ver Lampreia (2008). para a definição de subtipos de TEAs e para um melhor entendimento da relação existente entre a tríade de prejuízos que os define, mas, para isso, é preciso definir uma metodologia capaz de gerar os dados relevantes, o que irá depender do enfoque teórico adotado.

Originalmente, Kanner (1943) concebeu o autismo como um distúrbio do contato afetivo. Hoje o autismo é concebido como um desvio do desenvolvimento e, por essa razão, é classificado como um transtorno global do desenvolvimento (TGD), tanto na CID 10 quanto no DSM-IV-TR. Como visto, caracteriza-se por uma tríade de prejuízos nas áreas de interação social, comunicação e padrões restritos e repetitivos de comportamentos, interesses e atividades. Abrange um spectrum muito heterogêneo de quadros clínicos ou comportamentais, com diferentes graus de severidade: por exemplo, algumas crianças falam, outras não; algumas apresentam retardo mental, outras não. Wing e Gould (1979) classificaram as crianças autistas por elas estudadas em três grupos segundo o tipo de interação social apresentado: isolamento social, interação passiva e interação ativa, mas bizarra.

Os enfoques teóricos predominantes são a teoria da mente, de cunho inatista determinista, e a perspectiva desenvolvimentista, de cunho socioafetivo ${ }^{1}$. Hobson (2002) - importante representante desse último enfoque - considera que a falta de sensibilidade e responsividade afetiva do bebê que virá a ser diagnosticado como autista em relação a outro ser humano está na base do desvio do desenvolvimento, porém não o determina. Tendo nascido com falhas para o estabelecimento de engajamento afetivo, as interações sociais estarão em grande medida impossibilitadas, ou pelo menos prejudicadas, e, com isso, todo o seu desenvolvimento. Segundo esse enfoque, seria razoável supor que os marcadores do autismo - falta do apontar protodeclarativo, precursor da fala, e do jogo simbólico - que se desenvolvem na fase da intersubjetividade secundária por volta dos 9 meses de idade tivessem também seus precursores na fase anterior da intersubjetividade primária, como visto na introdução.

Essa perspectiva recomenda, assim, uma determinada metodologia de pesquisa para a identificação de sinais precoces de risco de autismo. Em primeiro lugar, seria fundamental observar interações de bebês de alto-risco, isto é, aqueles que têm um irmão com diagnóstico de autismo, com o seu cuidador. Essa observação deveria iniciar-se, se não antes, pelo menos entre 3 e 6 meses de idade, porque essa fase, até por volta dos 9 meses de idade, é caracterizada pela comunicação afetiva face a face ${ }^{2}$. Seria importante observar tanto categorias discretas de interação, como contato ocular, sorriso e vocalização, assim como categorias mais qualitativas de engajamento afetivo e fluxo da interação. Essas duas últimas podem ser extremamente relevantes, na medida em que bebês autistas, dependendo do grau de severidade do autismo, podem apresentar contato ocular ou sorrir, mas fazê-lo sem engajamento afetivo. 
Por fim, além da tríade de prejuízos que define o autismo, disfunções sensoriais também têm sido observadas em cerca de $70 \%$ dos autistas

(O’Neil \& Jones, 1997).
Se a lógica da perspectiva desenvolvimentista estiver correta, essas categorias afetivas deveriam permitir uma diferenciação entre bebês que receberão futuramente um diagnóstico dentro do espectro autístico e bebês que terão um desenvolvimento típico. Seria importante também que essas observações fossem regulares e próximas, isto é, de preferência semanais ou quinzenais, de maneira a acompanhar as mudanças de interação que ocorrem nesse período, ou a ausência delas. Isso caracterizaria um estudo mais propriamente longitudinal do que aqueles que têm sido realizados com bebês de alto risco, apresentados acima, que realizaram observações bem mais esparsas.

Passando à questão da identificação de subtipos de autismo, é preciso levar em conta a visão de que ele abrange um amplo espectro com diferentes graus de severidade. A classificação de Wing e Gould (1979) poderia permitir uma caracterização inicial do espectro, contudo, seria importante poder descrever, em termos longitudinais, as características iniciais e seu desenvolvimento, de maneira a melhor compreender em que consiste o desvio no desenvolvimento da criança autista, como, por exemplo, procurar identificar as características comuns das crianças que se tornaram mais isoladas, das que não adquiriram a fala e das que apresentaram retardo mental.

Esse tipo de descrição também poderia permitir melhor entendimento da relação entre os prejuízos na área de interação social, comunicação e interesses e comportamentos restritos e repetitivos, isto é, poderíamos, mediante esse agrupamento em diferentes subtipos e através de estudos longitudinais, melhor compreender a maneira como esses prejuízos estão interligados, se é um prejuízo na interação social que prejudica o desenvolvimento da comunicação e faz surgir os comportamentos e interesses estereotipados ou se os prejuízos na comunicação estão presentes também nas crianças que apresentam interação social.

Por fim, além da tríade de prejuízos que define o autismo, disfunções sensoriais também têm sido observadas em cerca de $70 \%$ dos autistas (O’Neil \& Jones, 1997). Por esse motivo, elas têm ganhado atenção crescente por parte dos pesquisadores, embora Kanner (1943) já fizesse menção a experiências sensoriais incomuns. A comunidade científica tem passado a valorizar os relatos autobiográficos de autistas de alto-funcionamento e a investigar o impacto das diferenças sensórioperceptivas no quadro clínico geral, e alguns estudos, como o de Baranek (1999), já citado, têm procurado investigar indícios de anormalidades nos primeiros meses de vida. Uma avaliação sensorial das crianças submetidas à metodologia de pesquisa acima descrita também deveria ser considerada. A identificação de disfunções sensoriais e sua correlação com diferentes quadros de autismo também poderia levantar hipóteses acerca de sua participação na sintomatologia.

Em suma, estudos longitudinais prospectivos que abranjam a observação quinzenal das interações diádicas do bebê de alto risco de autismo, a partir dos primeiros seis meses de vida, poderão iluminar algumas questões ainda sem respostas. 


\section{Carolina Lampreia}

Doutora em Psicologia clínica.

Professora assistente da Pontifícia Universidade Católica do Rio de Janeiro.

Endereço para envio de correspondência:

Departamento de Psicologia - PUC-Rio - Rua Marquês de São Vicente, 225. Cep: 22453-900

Rio de Janeiro - RJ.

E-mail: lampreia@puc-rio.br

Recebido 26/05/2008; Reformulado 06/01/2009; Aprovado 06/01/2009. and culture. In C. Moore \& P. J. Dunham (Eds.), Joint attention. Its origins and role in development (pp. 205-221). Hillsdale, NJ: Lawrence Erlbaum.

Adrien, J. L., Faure, M., Perrot, A., Hameury, L., Garreau, B., Barthélémy, et al. (1991). Autism and family home movies: Preliminary findings. Journal of Autism and Developmental Disorders, 21(1), 43-49.

Adrien, J. L., Lenoir, P., Martineau, J., Perrot, A., Hameury, L., Larmande, C., et al. (1993). Blind ratings of early symptoms of autism based upon family home movies. Journal of the American Academy of Child Adolescence Psychiatry, 32(3), 617-626.

American Psychiatric Association. (2002). DSM-IV-TR. Manual diagnóstico e estatístico de transtornos mentais (D. Batista, trad., 4a ed. rev.). Porto Alegre: Artes Médicas.

Baldwin, D. A. (1995). Understanding the link between joint attention and language. In C. Moore \& P. J. Dunham (Eds.), Joint attention. Its origins and role in development (pp. 131158). Hillsdale, NJ: Lawrence Erlbaum.

Baranek, G. T. (1999). Autism during infancy: A retrospective video analysis of sensory-motor and social behaviors at 9-12 months of age. Journal of Autism and Developmental Disorders, 29(3), 213-224.

Baron-Cohen, S., Allen, J., \& Gillberg, C. (1992). Can autism be detected at 18 months? The needle, the haystack, and the CHAT. British Journal of Psychiatry, 161, 839-843.

Bono, M. A., Daley, T., \& Sigman, M. (2004). Relations among joint attention, amount of intervention and language gain in autism. Journal of Autism and Developmental Disorders, 34(5), 495-505.
Bosa, C. (2002). Atenção compartilhada e identificação precoce do autismo. Psicologia: Reflexão e Crítica, 15(1), 77-88.

Bristol-Power, M. M., \& Spinella, G. (1999). Research on screening and diagnosis in autism: A work in progress. Journal of Autism and Developmental Disorders, 29(6), 435-438.

Bryson, S. E., Zwaigenbaum, L., Brian, J., Roberts, W., Szatmari, P., Rombough, V., et al. (2007). A prospective case series of high-risk infants who developed autism. Journal of Autism and Developmental Disorders, 37(1), 12-24.

Carpenter, M., Nagell, K., \& Tomasello, M. (1998). Social cognition, joint attention, and communicative competence from 9 to 15 months of age. Monographs of the Society for Research in Child Development, 63(4), 1-145

Carpenter, M., \& Tomasello, M. (2000). Joint attention, cultural learning, and language acquisition. Implications for children with autism. In A. M. Wetherby \& B. M. Prizant (Eds.), Autism spectrum disorders. A transactional developmental perspective (pp. 31-54). Baltimore, MA: Paul H. Brookes.

Cassel, T. D., Messinger, D. S., Ibanez, L. V., Haltigan, J. D., Acosta, S. I., \& Buchman, A. C. (2007). Early social and emotional communication in the infant siblings of children with autism spectrum disorders: An examination of the broad phenotype. Journal of Autism and Developmental Disorders, 37(1), 122-132.

Charman, T. (2004). Why is joint attention a pivotal skill in autism? In U. Frith \& E. L. Hill (Eds.), Autism: Mind and brain (pp. 6787). Oxford: Oxford University Press.

Charman, T., \& Baird, G. (2002). Practioner review: Diagnosis of autism spectrum disorder in 2- and 3-year-old children. Journal of Child Psychology and Psychiatry, 43(3), 289-305. 
Chawarska, K., Paul, R., Klin, A., Hannigen, S., Dichtel, L. E. \& Volkmar, F. (2007). Parental recognition of developmenta problems in toddlers with Autism Spectrum Disorders. Journal of Autism and Developmental Disorders, 37(1), 62-72.

Chawarska, K., \& Volkmar, F. R. (2005). Autism in infancy and early childhood. In F. R. Volkmar, R. Paul, A. Klin \& D. Cohen (Eds.), Handbook of autism and pervasive developmental disorders (Vol. 1, 3rd ed., pp. 223-246). Hoboken, NJ: John Wiley \& Sons.

Dawson, G., \& Galpert, L. (1986). A developmental model for facilitating the social behavior of autistic children. In E. Schopler \& G. B. Mesobov (Eds.), Social behavior in autism (pp. 237-261). New York: Plenum

Dawson, G., \& Lewy, A. (1989). Arousal, attention, and the socioemotional impairments of individuals with autism. In G. Dawson (Ed.), Autism: Nature, diagnosis and treatment (pp. 49-74). New York: Guildford.

Dawson, G., Munson, J., Estes, A., Osterling, J., McPartland, J., Toth, K., et al. (2002). Neurocognitive function and joint attention ability in young children with autism spectrum disorder versus developmental delay. Child Development, 73(2), 345-359.

Eaves, L. C., \& Ho, H. H. (2004). The very early identification of autism: Outcome to age $41 / 2-5$. Journal of Autism and Developmental Disorders, 34(4), 367-378.

Gamliel, I., Yirmiya, N., \& Sigman, M. (2007). The development of young siblings of children with autism from 4 to 54 months. Journal of Autism and Developmental Disorders, 37, 171-183.

Hobson, P. (2002). The cradle of thought. London: Macmillan.

Iverson, J. M., \& Wozniak, R. H. (2007). Variation in vocal-motor development in infant siblings of children with autism. Journal of Autism and Developmental Disorders, 37, 158-170.

Kanner, L. (1943). Autistic disturbances of affective contact. Nervous Child, 2, 217-250.

Lampreia, C. (2004). Os enfoques cognitivista e desenvolvimentista no autismo: uma análise preliminar. Psicologia, Reflexão e Crítica, 17, 111-120.

Lampreia, C. (2008). O processo de desenvolvimento rumo ao símbolo: uma perspectiva pragmática. Arquivos Brasileiros de Psicologia, 60(2), 117-128.

Lewis, M., \& Rosenblum, L. A. (1974). The effect of the infant on its caregiver. New York: John Wiley \& Sons.

Loh, L., Soman, T., Brian, J., Bryson, S. E., Roberts, W., Szatmari, P., et al. (2007). Stereotyped motor behaviors associated with autism in high-risk infants: A pilot videotape analysis of a sibling sample. Journal of Autism and Developmental Disorders, 37(1), 25-36.

Lord, C. (1995). Follow-up of two-year-olds referred for possible autism. Journal of Child Psychology and Psychiatry, 36(8), 1365-1382.

Lord C., Rutter M., \& Le Couteur, A. (1994). Autism diagnostic interview - revised: A revised version of a diagnostic interview for caregivers of individuals with possible pervasive developmental disorders. Journal of Autism and Developmental Disorders, 24, 659-685.

Merin, N., Young, G. S., Ozonoff, S., \& Rogers, S. J. (2007). Visual fixation patterns during reciprocal social interaction distinguish a subgroup of 6-months-old infants at-risk for autism from comparison infants. Journal of Autisn and Developmental Disorders, 379(1), 108-121.

O'Neil, M., \& Jones, R. S. P. (1997). Sensory-perceptual abnormalities in autism: A case for more research? Journal of Autism and Developmental Disorders, 27(3), 283-293.
Osterling J. A, \& Dawson, G. (1994). Early recognition of children with autism: A study of first birthday home videotapes. Journal of Autism and Developmental Disorders, 24(3), 247-257.

Osterling, J. A., Dawson, G., \& Munson, J. A. (2002). Early recognition of 1-year-old infants with autism spectrum disorder versus mental retardation. Development and Psychopathology, 14, 239-251.

Presnames, A. G., Walden, T. A., Stone, W. L., \& Yoder, P. J. (2007). Effects of different attentional cues on responding to joint attention in young siblings of children with autism spectrum disorders. Journal of Autism and Developmental Disorders, 37(1), 133-144.

Reznick, J. S., Baranek, G. T., Reavis, S., Watson, L. R., \& Crais, E. R. (2007). A parent-report instrument for identifying one-yearolds at risk for an eventual diagnosis of autism: The first year inventory. Journal of Autism and Developmental Disorders, 37(9), 1691-1710.

Robins, D. L., Fein, D., Barton, M. L., \& Green, J. A. (2001). The modified checklist for autism intoddlers: An initial study investigating the early detection of autism and pervasive developmental disorder. Journal of Autism and Developmental Disorders, 31(2), 131-143.

Schaffer, H. R. (1977). Studies in mother-infant interaction London: Academic Press.

Sigman, M., \& Kasari, C. (1995). Joint attention across contexts in normal and autistic children. In C. Moore \& P. J. Dunham (Eds.), Joint attention. Its origins and role in development (pp. 189-203). Hillsdale, NJ: Lawrence Erlbaum.

Stone, W. L., Coonrod, E. E., \& Ousley, O. Y. (2000). Brief report: Screening tool for autism in two-year-olds (STAT): Development and preliminary data. Journal of Autism and Developmental Disorders, 30(6), 607-612.

Stone, W. L., Lee, E. B., Ashford, L., Brissie, J., Hepburn, S. L., Coonrod, E. E., et al. (1999). Can autism be diagnosed accurately in children under 3 years? Journal of Child Psychology and Psychiatry, 40(2), 219-226.

Sullivan, M., Finelli, J., Marvin, A., Garrett-Mayer, E., Bauman M., \& Landa, R. (2007). Response to joint attention in toddlers at risk for autism spectrum disorder: A prospective study. Journal of Autism and Developmental Disorders, 37(1), 37-48.

Tomasello, M. (1995). Joint attention as social cognition. In C. Moore \& P. J. Dunham (Eds.), Joint attention. Its origins and role in development (pp. 103-130). Hillsdale, NJ: Lawrence Erlbaum.

Tomasello, M. (2003). Origens culturais da aquisição do conhecimento humano (C. Berliner, trad.). São Paulo: Martins Fontes.

Tomasello, M., \& Farrar, M. J. (1986). Joint attention and early language. Child Development, 57, 1454-1463.

Trevarthen, C., Aitken, K., Papoudi, D., \& Robarts, J. (1998). Children with autism. Diagnosis and interventions to meet their needs (2nd ed.). London: Jessica Kingsley.

Wetherby, A. M., Woods, J., Allen, L., Cleary, J., Dickison, H., \& Lord, C. (2004). Early indicators of autism spectrum disorders in the second year of life. Journal of Autism and Developmental Disorders, 34(5), 34, 473-493.

Wing, L., \& Gould, J. (1979). Severe impairments of social interaction and associated abnormalities in children: Epidemiology and classification. Journal of Autism and Developmental Disorders, 9(1), 11-29.

World Health Organization. (1998). International classification of diseases (10th rev.). Genebra: Author. 